\title{
METODOLOGIA PARA ESTUDO DE SELETIVIDADE DE HERBICIDAS NA CULTURA DA CANA-DE-AÇÚCAR (Saccharum spp). \\ Gilmar A. Montório ${ }^{1}$, Jamil Constantin ${ }^{2}$, Edivaldo D. Velini ${ }^{3}$, Thiago Montório ${ }^{4}$
}

${ }^{1} E^{2} g^{\circ}$. Agrônomo, Dr., Professor ESAPP - Paraguaçu Paulista / SP. montorio@ @etonne.com.br

${ }^{2}$ Eng ${ }^{\circ}$. Agrônomo, Dr., Professor Universidade Estadual de Maringá. Av. Colombo, 5790. Maringá, PR 87020-900

${ }^{3}$ Eng $^{\circ}$ Agrônomo, Dr., Professor FCA - UNESP, Botucatu, SP. C.P. 230 CEP 18603-970

${ }^{4}$ Acadêmico do Curso de Agronomia, Escola Superior de Agronomia de Paraguaçu Paulista -ESAPP

\begin{abstract}
RESUMO
Estudos sobre seletividade de herbicidas na cultura cana-de-açúcar, são feitos geralmente juntos com ensaios de eficácia sobre plantas daninhas e sempre utilizando-se uma testemunha por bloco experimental. Quando se analisa o contraste tratamento com herbicida em relação a uma única testemunha sem herbicida, há uma tendência em se obter maior valores para o coeficiente de variação, podendo dessa forma, ocultar os efeitos de alguns produtos não seletivos. O objetivo do presente trabalho, foi avaliar especificamente a seletividade de herbicidas sobre a produção de colmos da cana-de-açúcar, mantendo-se as parcelas constantemente capinadas, e utilizando-se duas testemunhas laterais adjacentes a cada tratamento com herbicida. Com essa metodologia, os resultados mostraram que os herbicidas 2,4-D + ametryn $(1,34+1,501$ i.a./ ha), tebuthiuron + diuron $(0,96+1,25$ l i.a/ ha $)$, 2,4-D + hexazinone + diuron $(1,34+0,27+0,941$ i.a./ ha), ametryn (1,50 1 i.a./ ha), tebuthiuron $(0,961$ i.a./ ha) e tebuthiuron + ametryn $(0,96+1,501$ i.a/ ha $)$ reduziram significativamente a produção de colmos em 19,5;17,0;16,6;15,1;11,9 e 10,9 t/ ha respectivamente, com níveis de significância menor que 3\%. Logo, tais produtos foram considerados não seletivos a cv. RB 835089 quando aplicados na pós-emergência da cultura.
\end{abstract}

Palavra chave: herbicidas, cana-de-açúcar, seletividade e produção

\section{METHODOLOGY FOR STUDY OF SELECTIVITY OF HERBICIDES IN THE CULTURE OF SUGAR-CANE (Saccharum spp).}

\begin{abstract}
Studies on herbicides selectivity in the culture sugar-cane, they plows generally done together with rehearsals of effectiveness on harmful plants and always being used the witness by experimental block. When the contrast treatment is analyzed with herbicide in relation to an only witness without herbicide, there is a tendency in obtaining larger values for the variation coefficient, being able to not in that way, to hide some non selective herbicides. Therefore, the objective of the present work, specifically went evaluate to herbicides selectivity about the production of stems of the sugar-cane, constantly staying the portions weeded, and being used two lateral witness adjacent to each treatment with herbicide. With that methodology, the results showed that the herbicides $2,4-\mathrm{D}+$ ametryn $(1,34+1,501$ i.a./ ha), tebuthiuron + diuron $(0,96+1,251 \mathrm{i} . \mathrm{a} / \mathrm{ha}), 2,4-\mathrm{D}+$ hexazinone + diuron $(1,34+0,27+$ 0,941 i.a./ ha), ametryn (1,50 1 i.a./ ha), tebuthiuron (0,96 1 i.a./ ha) and tebuthiuron + ametryn $(0,96+1,501 \mathrm{i} . \mathrm{a} / \mathrm{ha})$ they reduced the stems production significantly in 19,$5 ; 17,0 ; 16,6$; 15,$1 ; 11,9$ and $10,9 \mathrm{t}$ ha-1 respectively, with levels of smaller significance than $3 \%$. Therefore, such products were not considered selective the cv. RB 835089 when applied in the powder-emergency of the culture.
\end{abstract}

Key words: herbicides, sugar cane, selectivity and production 


\section{INTRODUÇÃOO}

Estudos sobre seletividade de herbicidas na cultura da cana-de-açúcar são feitos geralmente juntos com ensaios de eficácia agronômica nas plantas daninhas e observando-se os sintomas de injúrias visuais na cultura. Sabe-se que existem herbicidas que reduzem a produção de colmos sem apresentar efeitos visualmente detectáveis e também outros que provocam injúrias bastante acentuadas, mas permitem a cultura manifestar plenamente seu potencial produtivo (Velini et al. 1992; Lorenzi et al. 1994; Constantin,1996; Velini et al. 2000a). Diante do exposto, considera-se fundamental que sejam realizados estudos específicos para análisar os efeitos da fitointoxicação a cultura.

A maioria dos ensaios de seletividade, são conduzidos utilizando-se uma única testemunha dentro dos blocos experimentais. Dessa forma, quando se analisa o contraste tratamento com herbicida em relação a uma única testemunha sem herbicida, observa-se maiores valores para o coeficiente de variação. Essa análise estatística na maioria das vezes não consegue detectar significância, mesmo naqueles tratamentos que evidenciam reduções drásticas nos parâmetros analisados. Como consequiência, isso pode ocultar os efeitos de certos herbicidas considerados seletivos durante as avaliações (Sociedade Brasileira da Ciência das Plantas Daninhas, 1995).

O objetivo do presente trabalho foi avaliar a seletividade de herbicidas sobre a produção de colmos da cana-de-açúcar cv. RB 835089, aplicados na pré e pós-emergência da cultura, mantendo-se as parcelas constantemente capinadas e utilizando-se duas testemunhas laterais adjacentes a cada tratamento com herbicidas.

\section{MATERIAL E MÉTODOS:}

O experimento foi conduzido na safra 1996/1997 em área pertencente à Usina de Açúcar Santa Terezinha, localizada no distrito de Iguatemi, município de Maringá-PR, utilizando-se a cultivar RB 835089. Dentro de cada bloco foi distribuido 15 parcelas representando os 15 tratamentos em esquema de parcela sub-dividida, com 4 repetições, sendo as subparcelas compostas de cinco linhas de plantas com 10 metros de comprimento, espaçadas de $1,10 \mathrm{~m}$. Logo, a área total de cada subparcela foi de $55 \mathrm{~m}^{2}$, sendo considerado como área útil, as três linhas internas, totalizando portanto $33 \mathrm{~m}^{2}$. Para cada sub-parcela com herbicida foram utilizadas, duas testemunhas adjacentes localizadas nas laterais. Os herbicidas foram aplicados usando-se pulverizador costal pressurizado a $\mathrm{CO}_{2}$, provido de barra contendo quatro pontas de jato plano em leque, marca Teejet DG 110.04 VS, espaçadas entre si de $0,5 \mathrm{~m}$, com volume de calda de $250 \mathrm{l} / \mathrm{ha}$. Avaliou-se o efeito dos tratamentos com herbicida sobre o parâmetro produção de colmos, colhendo-se as 4 linhas centrais das unidades experimentais manualmente e pesando-as mediante uso de um dinamômetro. Posteriormente, esses dados foram transformados em $\mathrm{t} / \mathrm{ha}$. Os dados foram analisados de duas formas: no primeiro caso, para os contrastes entre pares de médias referentes aos tratamentos principais (herbicidas) e os tratamentos secundários (condição), utilizou-se o teste Tukey em nível de $5 \%$ de probabilidade. No segundo caso, foi feita a média de todas as testemunhas duplas dentro de cada repetição, como se houvesse uma única testemunha, como acontece nos delineamentos convencionais e aplicou-se teste Tukey a 5\% de probabilidade.

Para analisar o parâmetro produção de colmos de cana-de-açúcar, foram estabelecidos contrastes entre as médias das testemunhas e cada um dos demais tratamentos com herbicida, determinando-se o valor e o nível de significância da estatística "F", calculada para cada contraste.

O nível de significância correspondeu à probabilidade de incorrer-se em erro ao se admitir como diferente as médias consideradas.Também para o referido estudo, foi considerado herbicida seletivo aquele tratamento que apresentou nível de significância para os 
contrastes com probabilidade maior ou igual a 23\%. Quando os níveis de probabilidade estiveram próximos a $23 \%$, sugeriu-se repetição dos tratamentos para confirmação dos resultados.

\section{RESULTADOS E DISCUSSÃO}

Na Tabela 1 são apresentados os resultados de produtividade da cana-de-açúcar cv. RB 835089 obtidos em análise de variância onde as parcelas foram divididas em tratamento com herbicida e tratamento sem herbicida. Cada tratamento com herbicida foi analisado comparando-se seus resultados com a média de suas duas testemunhas laterais adjacentes.

Antes de optar por essa análise estatística, foram estudadas diversas outras opções de análise com o objetivo de encontrar aquela que mais se ajustasse à realidade dos dados da área experimental. Um exemplo dessas opções foi aquela onde os tratamentos com herbicidas são analisados em função da média das dezesseis testemunhas laterais $(15+1$ para fechar as 2 testemunhas/ tratamento), conforme se observa na Tabela 2. Nessa opção, verifica-se que os contrastes para tratamento com herbicida apresentaram níveis de probabilidade superiores aos observados na análise em parcelas subdivididas (Tabela 1). Por exemplo, o tratamento 2,4-D + ametryn $(1,34+1,501$ i.a./ ha) apresentou na análise de variância da Tabela 2 , significância entre os contrastes com nível de probabilidade de $57,90 \%$, evidenciando ser um tratamento seletivo para a cultura (valor maior que 23\%). Quando se analisa o mesmo tratamento em esquema de parcelas subdivididas (Tabela 1) encontra-se valor de 0,02\%. Como se determinou previamente que níveis de significância entre os contrastes com valores maiores que $23 \%$ seriam considerados herbicidas seletivos, o tratamento 2,4-D + ametryn pela Tabela 2 deveria ser então considerado seletivo (57,90\%). Entretanto esse comportamento não correspondeu ao observado na área experimental pois comparando-se a produção de colmos com a média de suas duas testemunhas laterais adjacentes, ficou evidenciado redução de 19,5 t/ há, portanto o tratamento foi considerado não seletivo para a cultura. Por essa razão, a opção que mais se aproximou da realidade experimental foi à análise de variância considerando-se a metodologia com parcelas subdivididas. Também por essa opção observouse menores valores para o coeficiente de variação, mostrando, assim maior precisão dos dados experimentais observados.

Utilizando a análise estatística que levou em consideração a presença de testemunhas laterais (Tabela 1), observou-se que todos os tratamentos aplicados na pré-emergência da cultura apresentaram níveis de significância com probabilidade maior que $23 \%(0,23)$. Logo, os herbicidas tebuthiuron ( 1,11 i.a./ ha); diuron (2,50 1 i.a./ ha); ametryn (2,25 1 i.a./ ha); hexazinone + diuron $(0,30+1,08 \mathrm{~kg}$ i.a/ ha) e oxyfluorfen $(0,72$ 1 i.a./ ha) foram considerados seletivos para o parâmetro produção de colmos, quando são aplicados na préemergência da cana-de-açúcar cv. RB 835089, conforme observado por Santos (1990), Lorenzi et al. (1994); Carvalho \& Pereira (1997); Rolim et al. (2000); Velini et al. (2000b); Dario et al. (2000); Dario \& Dario (2000); Azânia et al. (2001).

Por outro lado, quando os tratamentos com herbicidas foram aplicados na pósemergência da cultura, verificou-se reduções drásticas na produtividade de colmos, exceto para a mistura hexazinone + diuron $(0,27+0,94 \mathrm{~kg}$ i.a./ ha) e diuron $(1,251$ i.a./ ha $)$ aplicado isoladamente. Ainda nessa Tabela, observa-se que os herbicidas $2,4-\mathrm{D}+$ ametryn $(1,34+1,50$ 1 i.a./ ha); tebuthiuron + diuron $(0,96+1,251$ i.a./ ha); $2,4-\mathrm{D}+$ hexazinone + diuron $(1,34+$ $0,27+0,941$ i.a./ ha); ametryn (1,50 1 i.a./ ha); tebuthiuron $(0,961$ i.a/ ha) e tebuthiuron + ametryn $(0,96+1,50 \mathrm{~kg}$ i.a/ ha) apresentaram baixíssimos níveis de significância com probabilidade variando de $0,02 \%$ a $3,23 \%$. Tais valores estiveram associados a reduções significativas na produção final de colmos. Por exemplo, a mistura 2,4-D + ametryn reduziu 19,5 t/ ha de colmos quando o mesmo foi comparado com a média de suas testemunhas laterais; tebuthiuron + diuron reduziu 17,0 t/ ha; 2,4-D + hexazinone + diuron reduziu 16,7 t/ ha; ametryn, tebuthiuron e tebuthiuron + ametryn reduziram 15,1;12,0 e 10,9 t/ ha respectivamente. Tais tratamentos quando são instalados em ensaios utilizando-se uma única 
testemunha dentro do bloco experimental, tendem a ocultar a significância dos resultados e acabam erroneamente mostrando seletividade do produto para a cultura. Conforme observado, os herbicidas 2,4-D + ametryn $(1,34+1,501$ i.a./ ha); tebuthiuron + diuron $(0,96+1,251$ i.a/ ha); 2,4-D + hexazinone + diuron $(1,34+0,27+0,94 \mathrm{~kg}$ i.a/ ha); ametryn (1,50 1 i.a./ ha), tebuthiuron $(0,961$ i.a/ ha); tebuthiuron + ametryn $(0,96+1,501 \mathrm{i} . \mathrm{a} / \mathrm{ha})$ e diuron + ametryn $(1,25+1,501$ i.a./ ha) foram considerados não seletivos para o parâmetro produção de colmos, quando aplicados em pós-emergência da cana-de-açúcar cv. RB 835089, em contraste com a maioria dos estudos de seletividade realizado por diversos pesquisadores, que utilizaram em seus experimentos uma única testemunha sem herbicida dentro do bloco.

\section{CONCLUSÕES}

- Os herbicidas tebuthiuron (1,1 1/ ha); diuron (2,5 l/ ha); ametryn (2,25 1/ ha); hexazinone + diuron $(0,30+1,08 \mathrm{~kg} / \mathrm{ha})$ e oxyfluorfen $(0,72 \mathrm{l} / \mathrm{ha})$ foram considerados seletivos para o parâmetro produção de colmos quando aplicados na pré-emergência da cv. RB 835089;

- Os herbicidas 2,4-D+ametryn $(1,34+0,27+0,94 \mathrm{~kg} / \mathrm{ha})$; ametryn $(1,5 \mathrm{l} / \mathrm{ha})$; tebuthiuron $(0,96 \mathrm{l} / \mathrm{ha})$; tebuthiuron+ametryn $(0,96+1,5 \mathrm{l} / \mathrm{ha})$ e diuron+ametryn $(1,25+1,5 \mathrm{l} / \mathrm{ha})$ foram considerados não seletivos para o parâmetro produção de colmos quando aplicados na pós-emergência da cv. RB 835089;

- A utilização de testemunhas laterais adjacentes aos tratamentos com herbicidas se ajustou melhor à realidade da área experimental para ensaio de seletividade quando comparado a uma única testemunha dentro do bloco;

- Em ensaios específicos de seletividade de herbicidas na cultura da cana-de-açúcar é fundamental manter as unidades experimentais constantemente capinadas para que não sofra os efeitos da matocompetição, que pode mascarar os resultados.

- Também em ensaios especificos de seletividade é importante realizar a avaliação de produção final de colmos, não se limitando simplesmente aos sintomas de injurias visuais.

\section{REFERÊNCIAS BIBLIOGRÁFICAS}

AZÂNIA, C.A.M.; CASAGRANDE, A. A.; ROLIM, S.C. seletividade de imazapic às soqueiras de cana-de-açúcar (Saccharum spp). Planta Daninha, v.19,n.3,p.345-350,2001. 
CARVALHO, J.C. \& PEREIRA, W.S.P. Seletividade das misturas de oxyfluorfem e outros herbicidas à cana-soca cv. RB 72-454 em três estádios de crescimento. In: CONGRESSO BRASILEIRO DA CIÊNCIA DAS PLANTAS DANINHAS, 21., 1997, Caxambu. Resumos... Caxambu:SBCPD, 1997. p.252.

CONSTANTIN, J. Avaliação da seletividade do herbicida halosulfuron à cana-de-açúcar (Saccharum spp). Botucatu:UNESP, 1996. 71p. Tese de doutorado em Agronomia Faculdade de Ciências Agronômicas, Universidade Estadual Paulista, 1996.

DARIO, G.J.A. ; DARIO, P.W. Eficiência do herbicida oxadiargyl no controle de plantas daninhas em soqueira de cana-de-açúcar. In: CONGRESSO BRASILEIRO DA CIÊNCIA DE PLANTAS DANINHAS, 22., 2000, Foz do Iguaçu.Resumos... Foz do Iguaçu:SBCPD, 2000. p.308.

DARIO, G.J.A.; DELLA VALLE, J.N.; DELLA VALLE, F.N. Eficiencia de herbicidas no controle de plantas daninhas na cultura da cana-de-açúcar. In: CONGRESSO BRASILEIRO DA CIÊNCIA DE PLANTAS DANINHAS, 22., 2000, Foz do Iguaçu. Resumos... Foz do Iguaçu:SBCPD, 2000. p.305.

LORENZI, H. J.; BRUNELLI NETO, V.; OLIVEIRA, J.E. Estudo do efeito do herbicida oxyfluorfen, aplicado em pré-emergência, sobre o crescimento e produtividade da cana-deaçúcar (Saccharum officinarum) cv. SP 71-6163. STAB, Piracicaba, v.12, p.25-26, 1994.

ROLIM, J.C.; JANEGITZ, I.; GARMS, M.A. Tolerância de variedades de cana-de-açúcar a herbicidas 1 - cana planta, solo arenoso, em pré-emergência. In: CONGRESSO BRASILEIRO DA CIENCIA DAS PLANTAS DANINHAS, 22., Foz do Iguaçu. Resumos... Foz do Iguaçu: SBCPD, 2000. p. 294.

SANTOS, A. J.S. Sensibilidade de variedades de cana-de-açúcar a herbicidas aplicados em três diferentes épocas. Piracicaba: USP, 1990. 80p. Dissertação de Mestrado em Fitotecnia - ESALQ, Universidade de São Paulo, 1990.

SOCIEDADE BRASILEIRA DA CIÊNCIA DAS PLANTAS DANINHAS. Procedimentos para instalação, avaliação e análise de experimentos com herbicidas. Londrina: SBCPD, 1995. 42p.

VELINI, E.D.; MARTINS, D.; MANOEL, L.A.; MATSUOKA, S.; TRAVAIN, J.C.; CARVALHO, J.C. Avaliação da seletividade de oyfluorfen e ametryn aplicado em pré ou pós-emergência, a dez variedades de cana-de-açúcar. Planta Daninha, v.18, n.1, p.123-134, $2000^{\text {a }}$

VELINI, E.D.; FREDERICO, L.A.; MORELLI, J.L.; MARUBAIASHI, O. M. Avaliação dos efeitos de doses do herbicida clomazone aplicado em pós-emergência inicial sobre o crescimento e produtividade de soqueira de cana-de-açúcar. STAB, v.10, p. 13-16, 1992.

VELINI, E. D.; PAGGIARO, C. M.; PEREIRA, W. S. P. Seletividade de Goal 240 CE, aplicado em pós-emergência, a 10 variedades de cana-de-açúcar (cana-soca). In: CONGRESSO BRASILEIRO DE CIÊNCIA DAS PLANTAS DANINHAS, 22., 2000, Foz do Iguaçu. Resumos... Foz do Iguaçu: SBCPD, 2000 b. p. 298. 
Tabela 1 -Média dos contrastes da produção de colmos (ton/ ha), valores de probabilidade "F", coeficientes de variações e diferenças de produção em \% e em t/ ha entre os tratamento com e sem herbicida do experimento realizado na Fazenda Santa Maria - Usina de Açúcar Santa Terezinha, Maringa-PR, 1997.

\begin{tabular}{|c|c|c|c|c|c|c|}
\hline \multirow{2}{*}{ Tratamentos } & \multirow{2}{*}{$\begin{array}{l}\text { Modo de } \\
\text { Aplicação }\end{array}$} & \multirow{2}{*}{ CONDIÇÃO } & \multicolumn{2}{|c|}{ Produção de colmos } & \multicolumn{2}{|c|}{ Difer. Com x Sem } \\
\hline & & & ton/ ha & $(\mathrm{Pr}>\mathrm{F})$ & $\%$ & (ton/ ha) \\
\hline Tebuthiuron & pré-emerg. & $\begin{array}{l}\text { SEM } \\
\text { COM }\end{array}$ & $\begin{array}{l}89,34 \\
78,50\end{array}$ & $\begin{array}{c}* \\
0,7079\end{array}$ & (2) & $(1,8)^{\star \star \star}$ \\
\hline Diuron & pré-emerg. & $\begin{array}{l}\text { SEM } \\
\text { COM }\end{array}$ & $\begin{array}{c}89,2 \\
88,63\end{array}$ & 0,9077 & $(0,6)$ & $(0,5)$ \\
\hline Ametryn & pré-emerg. & $\begin{array}{l}\text { SEM } \\
\text { COM }\end{array}$ & $\begin{array}{l}85,51 \\
85,79\end{array}$ & 0,9537 & $(0,4)$ & $(0,3)$ \\
\hline Hexazinone + Diuron & pré-emerg. & $\begin{array}{l}\text { SEM } \\
\text { COM }\end{array}$ & $\begin{array}{l}92,75 \\
89,49\end{array}$ & 0,5074 & $(3,5)$ & $(3,3)$ \\
\hline Oxyfluorfen & pré-emerg. & $\begin{array}{l}\text { SEM } \\
\text { COM }\end{array}$ & $\begin{array}{l}93,61 \\
93,46\end{array}$ & 0,9769 & $(0,2)$ & $(0,2)$ \\
\hline Tebuthiuron + Diuron & pós-emerg. & $\begin{array}{l}\text { SEM } \\
\text { COM }\end{array}$ & $\begin{array}{l}91,19 \\
74,15\end{array}$ & 0,0011 & $(18,6)$ & (17) \\
\hline Tebuthiuron + Ametrina & pós-emerg. & $\begin{array}{l}\text { SEM } \\
\text { COM }\end{array}$ & $\begin{array}{l}83,52 \\
72,73\end{array}$ & 0,0323 & (13) & $(10,9)$ \\
\hline Diuron + Ametrina & pós-emerg. & $\begin{array}{l}\text { SEM } \\
\text { COM }\end{array}$ & $\begin{array}{l}89,91 \\
84,37\end{array}$ & 0,2629 & $(6,2)$ & $(5,6)$ \\
\hline 2,4-D + Ametrina & pós-emerg. & $\begin{array}{l}\text { SEM } \\
\text { COM }\end{array}$ & $\begin{array}{c}105,54 \\
86,08\end{array}$ & 0,0002 & $(18,4)$ & $(19,5)$ \\
\hline 2,4-D + Hexazinone + Diuron & pós-emerg. & $\begin{array}{l}\text { SEM } \\
\text { COM }\end{array}$ & $\begin{array}{l}84,23 \\
67,61\end{array}$ & 0,0014 & $(19,8)$ & $(16,7)$ \\
\hline Ametryn & pós-emerg. & $\begin{array}{l}\text { SEM } \\
\text { COM }\end{array}$ & $\begin{array}{l}92,33 \\
77,27\end{array}$ & 0,0035 & $(16,3)$ & $(15,1)$ \\
\hline Hexazinone + Diuron & pós-emerg. & $\begin{array}{l}\text { SEM } \\
\text { COM }\end{array}$ & $\begin{array}{l}77,27 \\
77,84\end{array}$ & 0,9073 & 0,5 & 0,4 \\
\hline Tebuthiuron & pós-emerg. & $\begin{array}{l}\text { SEM } \\
\text { COM }\end{array}$ & $\begin{array}{l}91,19 \\
79,26\end{array}$ & 0,0186 & $(13,2)$ & $(12)$ \\
\hline Diuron & pós-emerg. & $\begin{array}{l}\text { SEM } \\
\text { COM }\end{array}$ & $\begin{array}{l}85,94 \\
82,38\end{array}$ & 0,4710 & $(4,2)$ & $(3,6)$ \\
\hline $2,4-D$ & pós-emerg. & $\begin{array}{l}\text { SEM } \\
\text { COM }\end{array}$ & $\begin{array}{l}89,63 \\
83,81 \\
\end{array}$ & 0,2397 & $(6,5)$ & $(5,9)$ \\
\hline
\end{tabular}

\begin{tabular}{lrr}
\hline \multicolumn{1}{c}{ Valores de F: ${ }^{* *}$} & & \\
\hline Herbicidas (H) & 1,21 & \\
Blocos (BL) & 22,94 & 0,3019 \\
Hx BL & 4,4 & 0,0001 \\
C.V. (\%) & 16,9 & 0,0001 \\
\hline Condição & 34,27 & \\
C x H & 2,11 & 0,0001 \\
C. V. (\%) & 8,06 & 0,0298 \\
\hline
\end{tabular}

* Níveis a que foram significativos os contrastes envolvendo as condições SEM e COM herbicidas avaliados na colheita.

** Níveis de probabilidade que foram significativos aos efeitos de blocos e tratamentos.

$\mathrm{Pr}=$ Probabilidade de significância para os contrastes envolvendo as médias verificadas em cada condição.

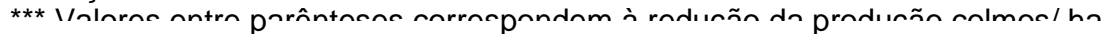


Tabela 2 - Média dos contrastes, valores de probabilidade de F e coeficientes de variação obtidos quando os tratamentos herbicidas, são analisados em função da média das 15 testemunhas sem herbicida para dados de produção de colmos obtidos durante a colheita do experimento na Fazenda Santa Maria - Usina de Açúcar Santa Terezinha, Maringá-PR, 1997.

\begin{tabular}{|c|c|c|c|}
\hline Tratamentos & $\begin{array}{c}\text { Dose } \\
\mathrm{kg}, \text { L i.a. ha }\end{array}$ & Aplicação & $\begin{array}{c}\text { Produção de colmos } \\
(\operatorname{Pr}>\mathrm{F})\end{array}$ \\
\hline Tebuthiuron & 1,1 & pré & * 0,7306 \\
\hline Diuron & 2,5 & pré & 0,8599 \\
\hline Ametryn & 2,25 & pré & 0,5506 \\
\hline Hexazinone + Diuron & $0,33+1,12$ & pré & 0,9603 \\
\hline Oxyfluorfen & 0,72 & pré & 0,5898 \\
\hline Tebuthiuron + Diuron & $0,86+1,25$ & pós & 0,0220 \\
\hline Tebuthiuron + Ametryn & $0,96+1,25$ & pós & 0,0127 \\
\hline Diuron + Ametryn & $1,5+1,5$ & pós & 0,4199 \\
\hline 2,4-D + Ametryn & $1,61+1,50$ & pós & 0,5790 \\
\hline 2,4-D + Hexazinone + Diuron & $1,61+0,28+0,98$ & pós & 0,0014 \\
\hline Ametryn & 1,5 & pós & 0,0653 \\
\hline Hexazinone + Diuron & $0,28+0,98$ & pós & 0,3513 \\
\hline Tebuthiuron & 0,96 & pós & 0,1199 \\
\hline Diuron & 1,25 & pós & 0,2716 \\
\hline 2,4-D & 1,61 & pós & 0,3734 \\
\hline Valores de $\mathrm{F}$ & & ** & \\
\hline Tratamentos & & 0,3476 & \\
\hline CV (\%) & & 15,13489 & \\
\hline
\end{tabular}

* Níveis que foram significativos os contrastes envolvendo as 16 testemunhas sem herbicidas.

${ }^{* *}$ Níveis de probabilidade que foram significativos os efeitos de blocos e tratamentos. 